\title{
Monte Carlo Evaluation of Single Event Effects in a Deep-Submicron Bulk Technology: Comparison Between Atmospheric and Accelerator Environment
}

\author{
Angelo Infantino, Rubén García Alía, and Markus Brugger
}

\begin{abstract}
In this work, the expected SEE rate in a generic model of a state-of-the-art SRAM memory was studied as a function of the critical charge in different radiation environments. The FLUKA Monte Carlo code was used to evaluate the SEE mono-energetic cross section for different particles as well as the SEE rate in different mixed field environments through an energy deposition analysis. Mono-energetic cross sections for protons and both positively and negatively charged muons were evaluated in the 0.1-1000 $\mathrm{MeV}$ energy range. The SEE rate was calculated for different mixed radiation field environments such as the terrestrial environment, commercial flight altitude, LHC critical areas for electronics and CERN's CHARM test facility. Results show that direct ionization from charged particles becomes predominant between 0.2-1.1 fC compared to indirect neutron energy deposition. Finally the CHARM facility was demonstrated to be able to reproduce with a good approximation both the atmospheric and accelerator environments, particularly with regard to avionics, ground level applications and LHC.
\end{abstract}

Index Terms-Accelerator, CERN, CHARM, FLUKA, galactic cosmic rays (GCR), ground level applications, large hadron collider (LHC), Monte Carlo method, single event effects (SEE).

\section{INTRODUCTION}

$\mathbf{T}$ HE composition of terrestrial ionizing radiation is largely due to the particle shower of primary cosmic rays (protons, helium ions and heavier ions) in the Earth's atmosphere: after several generations of interactions, the sea level environment consists of a mixture of neutrons, protons, pions, muons and other particle species [1]. In recent years, there was an increasing interest in the assessment and modelling of Galactic Cosmic Rays (GCR) particularly regarding the evaluation of the radiation effects on airline crew and passengers, interplanetary missions and on-board microelectronics.

Radiation effects in electronic devices can be divided into two main categories: cumulative effects and Single Event Effects (SEE). The risk of failure of the aforementioned SEEs

Manuscript received July 8, 2016; revised September 14, 2016; accepted October 20, 2016. Date of publication October 25, 2016; date of current version February 28, 2017.

The authors are with the European Organization for Nuclear Research (CERN), 1211 Geneva 23, Switzerland (e-mail: angelo.infantino@ cern.ch; ruben.garcia.alia@cern.ch; markus.brugger@cern.ch).

Color versions of one or more of the figures in this paper are available online at http://ieeexplore.iee.org.

Digital Object Identifier 10.1109/TNS.2016.2621238 becomes high as electronic components (e.g. logic, power devices) are present in a growing number of equipment, such in avionics, space applications and complex accelerator facilities. Indeed, today the problem is not just limited to SEE as used in avionics [2], but are also more and more observed at ground level [3]. Furthermore, the continued advance in fabrication of technologies toward smaller integrated circuit feature sizes, the higher speeds and more complex circuitry increase the sensitivity to SEE, including terrestrial errors in a benign desktop environment, and may also lead to new failure mechanisms [4].

In literature, the effects of galactic cosmic neutrons are well documented [3], [5]-[6] while the effects of other kinds of particles, particularly muons, were investigated only in a few publications [7]-[10]. The need to investigate the SEE beyond the "classical" vision that only certain kind radiation fields may have a significant impact on the technology currently available, was considered in [10]. QARM [11], [12] and EXPACS [13], [14] codes were extensively used in the past [10] to assess particle spectra in the atmospheric and terrestrial environments, but a systematic evaluation of the different particle contribution to the total SEE rate, in a given environment, has been investigated only in a few publications [15]. In [15] spectra from QARM and EXPACS were used as primary source to investigate the impact of terrestrial radiation on soft error. Recently, our group demonstrated how FLUKA Monte Carlo simulation allows one to assess the environmental particle flux distribution, particularly of atmospheric and terrestrial muons, down to low energies and with a better statistics compared to the codes reported above [16].

On the other hand, in the accelerator field a detailed modelling and an accurate experimental measurement of these effects become important to prevent undesired shutdowns and maintenances in complex accelerator facilities. In the past, a considerable amount of work has been done at CERN in Radiation to Electronics (R2E) oriented studies for the Large Hadron Collider (LHC) [17]-[19]. Indeed, LHC needs a significant amount of electronic components to control and monitor the various infrastructures and systems. In the LHC tunnel, the electronic equipment is installed in dedicated alcoves, typically labelled UJ or RR, and separated from the beam line by various configurations of concrete and/or iron shielding walls. 
The UJ areas are, compared to the RR, heavily shielded and house a large number of electronics which is critical for the machine operation and protection [17].

The comparison between the environmental and the accelerator field is important in the design of new test facilities able to reproduce, with a good approximation, a mixed radiation field needed to test new integrated technologies for special applications, such as avionics and space environment. Indeed, in order to test specific electronic equipment in a radiation field similar to the one occurring at CERN accelerators (e.g. in the LHC) and to study the respective equipment sensitivity to assure mid/long-term operation requirements, a new irradiation facility called CHARM has been built within the CERN site and fully in operation since 2014. This facility is not only useful for testing devices within accelerator representative environments, but its available radiation fields are also characteristic of ground and atmospheric environments as well as the space environment [20], [21].

In this context, Monte Carlo codes are useful tools to use in assessing both particle spectra, SEE cross sections and rates. Moreover the use of validated models [16], in terms of physical and transport parameters to be applied, become strategic in planning new accelerator facilities, in the design of new integrated technologies and in the optimization of testing campaigns.

In this work, the FLUKA [22], [23] Monte Carlo code was used to show how the classical vision that only certain kind radiation fields may have a significant impact on the technology currently available is outdated. Furthermore, the SEE rate in a general model of a state-of-the-art SRAM memory was evaluated as a function of the critical charge in different radiation environments such as the terrestrial environment, commercial flight altitude, the LHC critical areas for electronics and the CHARM facility. Atmospheric and terrestrial neutron, proton and muon spectra, previously assessed and validated by our group, were used as a primary source of a simplified FLUKA model of a SRAM memory. The contribution from different particle to the total SEE rate was investigated. In each case, the critical charge where the transition between indirect-direct ionization dominance of the overall SEE rate was calculated. The SEE rate at the LHC point 7 (IR7) and the CHARM facility was evaluated in critical areas for the electronics and in predefined test locations respectively. Finally, the results obtained in the accelerator case were compared with the environmental one. For a complete understanding of the phenomena involved, mono-energetic cross sections for protons and muons were evaluated at different energies in addition to the mixed field environment.

\section{FLUKA CAPABILITy in Modelling GCR}

Recently, our group demonstrated the capability of FLUKA in modelling GCR with high accuracy and down to lower energy [16] compared to other available tools, such as QARM and EXPACS. A brief summary of the main achievements in [16] is reported in the following as it represents the validation of the environmental particle spectra used in this work. Indeed, in [16] we assessed different particle spectra in an

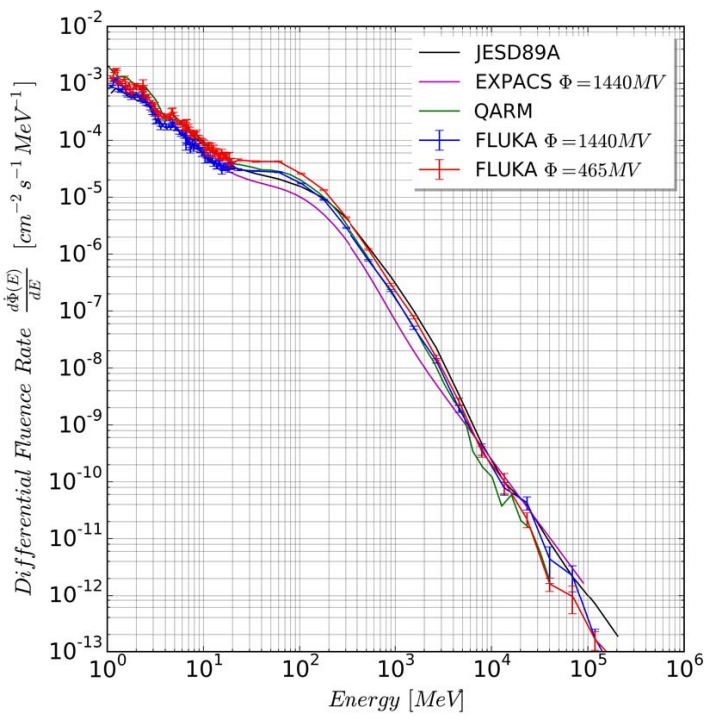

Fig. 1. Absolute comparison of FLUKA differential neutron fluence rate distributions in energy (NYC, ground level, solar maximum and minimum) against the JESD89A standard, QARM (mid solar activity) and EXPACS (solar maximum) codes.

atmospheric and terrestrial environment and we validated them through a comparison with well-known reference available in literature, two of the most used tool for the assessment of atmospheric particle spectra and experimental measurements conduct at TRIUMF. The details of the validation can be found in [16] while for the purpose of the present work only the main achievements are summarized. The already validated particle spectra, will be applied in the present work as the primary source term and transported through a generic model of a SRAM memory.

In [16] FLUKA was used to assess the atmospheric and terrestrial neutron and muon differential fluxes at various altitudes and specific examples such as the geographic coordinates corresponding to Vancouver and New York City (NYC). The latter, was used as reference case for the validation of our model in terms of physics and transport parameters to be applied: particle spectra, assessed for two different solar modulation values (referred in what follows as "solar maximum" for $\Phi=1440 \mathrm{MV}$ and "solar minimum" for $\Phi=465 \mathrm{MV}$ ), were compared with references available in literature, i.e. JEDEC standard No. 89A [5], as well as the output from the QARM and EXPACS codes. The comparison of the differential neutron fluence rate is reported in Fig. 1. Fig. 1 shows an overall good agreement, in a wide energy range, between FLUKA simulations and the experimental differential neutron flux reported in the JESD89A standard: considering two reference energies of $1 \mathrm{MeV}$ and $100 \mathrm{MeV}$, the agreement is within $30 \%$ and $14 \%$ respectively. With regard to EXPACS and QARM the agreement is globally within $56 \%$ and $33 \%$ respectively. Fig. 2 shows the comparison of the differential muon fluence rate assessed using FLUKA, EXPACS and QARM: at the reference energy of $1 \mathrm{MeV}$ (zoom window in Fig. 2) EXPACS underestimates the differential fluence rate by a factor $\sim 3$; with regard to QARM, the agreement is greater than a factor 20 due to the high statistical fluctuation. 


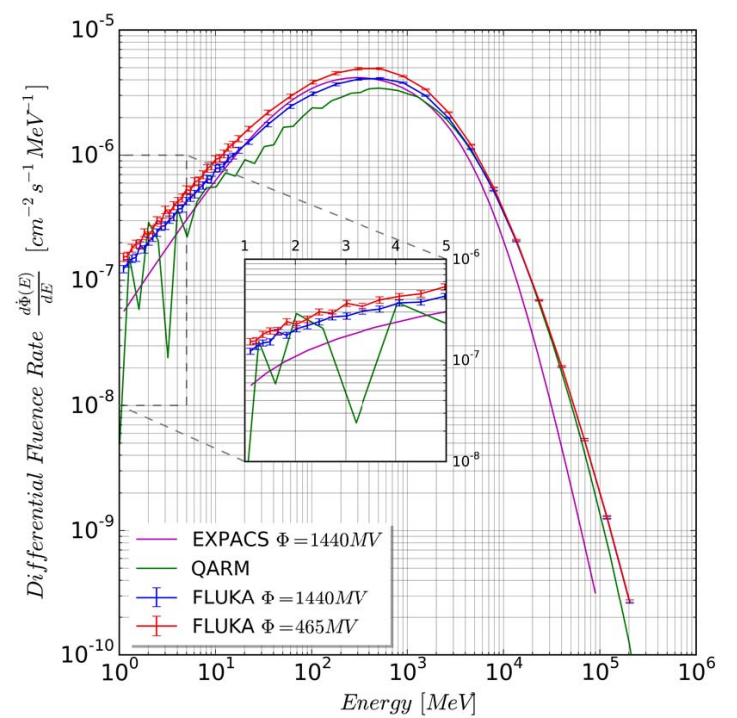

Fig. 2. Absolute comparison of FLUKA differential muon fluence rate distributions in energy (NYC, ground level, solar maximum and minimum) against QARM (mid solar activity) and EXPACS (solar maximum) codes.

\section{MATERIALS AND METhods}

\section{A. Geometry of a Generic $45 \mathrm{~nm}$ SRAM Memory}

SEE rates in different radiation environments and mono-energetic cross sections were evaluated using FLUKA Monte Carlo simulation. In this work, we used a general RPP model of a state-of-the-art SRAM memory, based on a previous model created and benchmarked in the past from our group [24], to score the event-by-event energy deposition in the sensitive volume. The model of the SRAM memory is based on what is reported in [25]. In order to simulate a general commercial $45 \mathrm{~nm}$ SRAM technology, a $6.5 \mu \mathrm{m}$ backend-of-line (BEOL) zone consisting of silicon dioxide and aluminum layers was placed above the sensitive volume (SV) region. To include the effect of the secondary particles arriving from the materials surrounding the SV, while preserving a reasonable statistical precision, 400 different collection volumes $(\mathrm{CV}$, i.e. the volumes where the deposited energy is scored), were created and placed on the same plane perpendicular to the beam direction (z-direction) in the centre of a $50 \mu \mathrm{m} \times$ $50 \mu \mathrm{m}$ surface. The replication of the $\mathrm{CV}$ allows to increase the simulation statistics while preserving the effect of secondary particles coming from the sides. The CV size was $300 \mathrm{~nm} \times 300 \mathrm{~nm} \times 500 \mathrm{~nm}$ [25]. Fig. 3 shows a vertical (plane $\mathrm{ZY}$ ) and horizontal (plane $\mathrm{XY}$ ) cut of the simulated geometry.

\section{B. Source particle spectra}

A rectangular shaped beam $50 \mu \mathrm{m} \times 50 \mu \mathrm{m}$ was simulated and directed through the SRAM model as indicated in Fig. 3.

In the mono-energetic study, the beam energy was set in order to uniformly cover the energy range $0.1-1000 \mathrm{MeV}$. The mono-energetic cross sections were evaluated for protons, $\mu^{ \pm}$, and additionally for $100 \mathrm{keV}$ electrons.

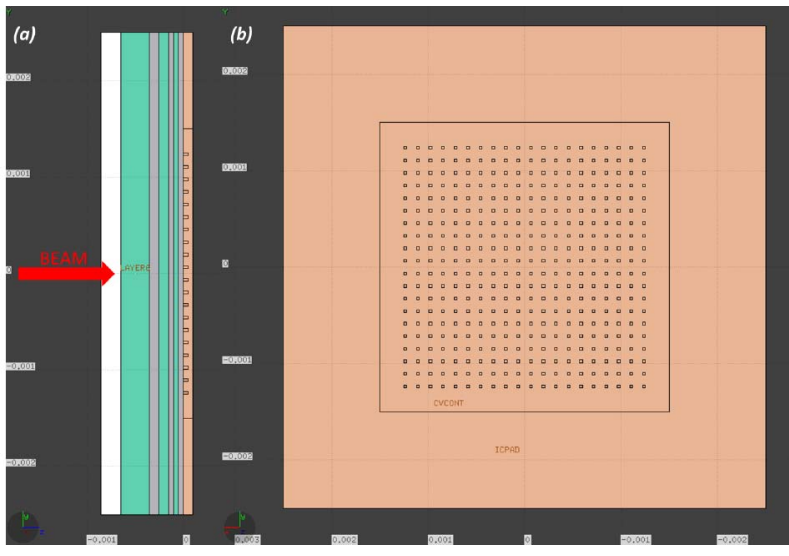

Fig. 3. Cut in the ZY (a) and XY (b) plane of the FLUKA model used in the simulations. The beam travels in the positive $\mathrm{z}$ direction. From left to right of Fig. 3a the silicon dioxide (green) and aluminum (grey) layers can be observed, representing the BEOL; then the silicon region in which the CVs are placed can be seen. In Fig. $3 \mathrm{~b}$ is possible to see the $400 \mathrm{CV}$ s modelled to improve the statistics of the simulation.

With regard to the mixed particle energy spectra study, different sets of particle spectra were used as primary particle and simulated through a dedicated source routine:

I Environmental particle spectra. Atmospheric and terrestrial neutron, proton, $\mu^{ \pm}$spectra were used. The spectra were evaluated through the FLUKA Galactic Cosmic Rays (GCR) simulations summarized in section II and benchmarked, against reference data [5] and experimental measurements [26]. Spectra were evaluated in the area of Vancouver ( $49^{\circ} 15^{\prime} 00^{\prime \prime} \mathrm{N} 123^{\circ} 8,0$ " W) in the $1 \mathrm{keV}-10 \mathrm{TeV}$ energy range $\left(10^{-5} \mathrm{eV}-10 \mathrm{TeV}\right.$ for neutron) at different atmospheric depths $\left(1033 \mathrm{~g} / \mathrm{cm}^{2}\right.$, $909 \mathrm{~g} / \mathrm{cm}^{2}$ and $211 \mathrm{~g} / \mathrm{cm}^{2}$ ). Two shielding configurations at ground level, $1 \mathrm{~m}$ and $10 \mathrm{~m}$ thick of concrete (labelled light and heavy shielding in the following), were studied to simulate the generic structure of a building/heavy shielding [16].

II LHC point 7. LHC IR7 was chosen as reference study case for the accelerator field. In particular, particle spectra at the UJ and RR alcove, previously assessed in [19], [27] using FLUKA, were used: the neutron, proton, $\pi^{ \pm}$and $\mu^{ \pm}$differential fluence distribution were assessed for two reference locations where the electronics is placed in the $10^{-3}-10^{4} \mathrm{GeV}\left(2 \cdot 10^{-2}-10^{3} \mathrm{GeV}\right.$ for neutrons) energy range. UJ areas allow for more protection of the electronic racks due to the thickness and configuration of the shielding: indeed, the High Energy Hadron (HEH) fluence, i.e. hadrons $>20 \mathrm{MeV}$, is typically larger in these areas due to the vicinity to the radiation source. RR areas (light shielded) are typically shielded with $40+40 \mathrm{~cm}$ of iron and concrete respectively; UJ areas (heavily shielded) are typically shielded with $40+200 \mathrm{~cm}$ of iron and concrete respectively.

III CHARM. Three test locations were chosen as reference cases: test locations $1 \quad\left(\sim 90^{\circ}\right.$ from target $)$ and $6\left(\sim 45^{\circ}\right.$ from target) are shielded with $40 \mathrm{~cm}$ of iron $+40 \mathrm{~cm}$ of concrete; test location 11 is placed 
downstream the target selector without any shielding. Particle spectra of protons, $\mu^{ \pm}, \pi^{ \pm}$, neutrons were previously assessed using a FLUKA model of the full facility, developed and validated by our group [20], in the $10^{-6}-10^{2} \mathrm{GeV}$ energy range with a logarithmic binning (from thermal energy for neutrons).

\section{Physics and Scoring}

The PRECISIO default, a pre-defined set of transport thresholds and physical mechanism enabled, was chosen as basis for the physical and transport parameters of the simulation. However, to allow for a more accurate particle transport, some of them were overwritten: in particular, the proton, $\mu^{ \pm}, \pi^{ \pm}$ and electron transport threshold, the delta-ray and the electron production threshold was set to $1 \mathrm{keV}$; and COALESCE and EVAPORAT physics cards were enabled [23].

The event-by-event energy deposition distribution was scored for each case using a set of customized routines: the energy deposition was scored in the $10^{-4}-10^{2} \mathrm{MeV}$ energy range divided in 144 logarithmic bins. The integral of the deposition events above a certain critical charge $Q_{\text {crit }}$ (assuming $3.6 \mathrm{eV}$ [28] needed to generate a free charge carrier) were calculated, divided by the simulated fluence and conveniently normalized providing the SEE rate, as a function of $Q_{c r i t}$, expressed in FIT/Mbit ( 1 FIT $=1$ Failure in $10^{9}$ device-hours of operation). It is important to mention that in the following we will generally refer to SEE given that our FLUKA model scored the energy deposition in the sensitive volume without considering any specific effects on the SRAM memory. Nevertheless, considering the model that we implemented, the dimensions of the sensitive volume and the critical charges presented in the results, a Single Event Upset (SEU), i.e. a bit flip between two logical states, is the most important effect to take into account.

\section{Results}

Fig. 4 shows the SEE cross section, expressed in $\mathrm{cm}^{2} \mathrm{bit}^{-1}$, as a function of the critical charge for different mono-energetic particle beams.

For a better understanding, only some reference cases are reported in the plot. High and low energy particles were compared to emphasize the different interaction modes. With regard to protons, it is possible to see how high energy protons may interact with the sensitive volume both through direct and indirect ionization; on the other hand, low energy protons interact only through direct ionization and their contribution become significant only below a few fC. It is important to note how the curve for low energy electrons and high energy protons have a similar shape below $\sim 0.3-0.4 \mathrm{fC}$ meaning that components that are sensitive to low energy electrons [29] may also be sensitive to direct ionization by high energy protons. With regard to muons, both positively and negatively charged particle have the same trend at high energy while, at low energy, negative muon capture has a significant contribution.

From the curves reported in Fig. 4, and considering all the energies and particles simulated, it is possible to build the SEE cross section as a function of the incident energy

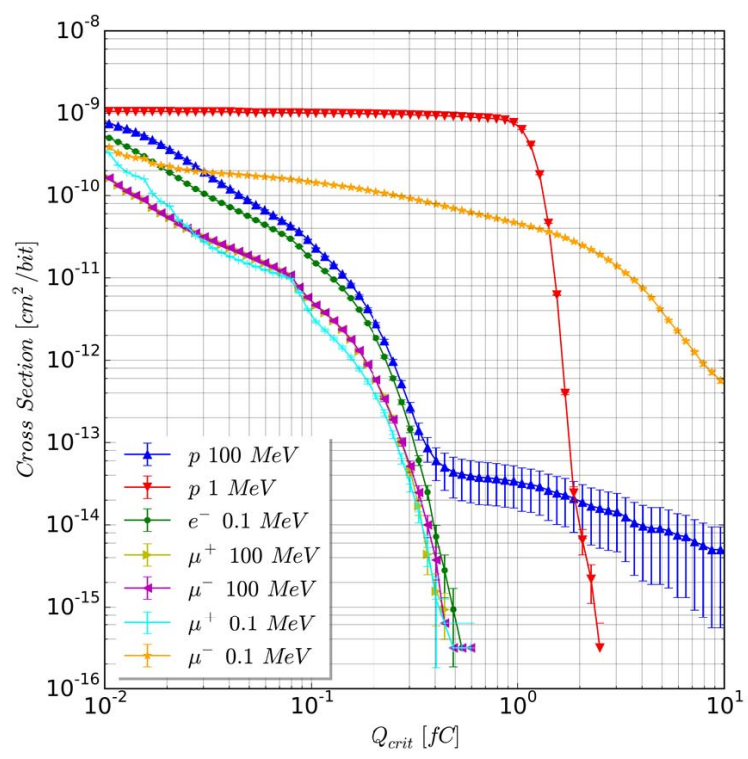

Fig. 4. Evaluation of the SEE cross section, as a function of the critical charge, for mono-energetic particle beams. For a better understanding, only some reference cases are reported in the plot, namely high and low energy protons, $\mu^{ \pm}$and $0.1 \mathrm{MeV}$ electrons.

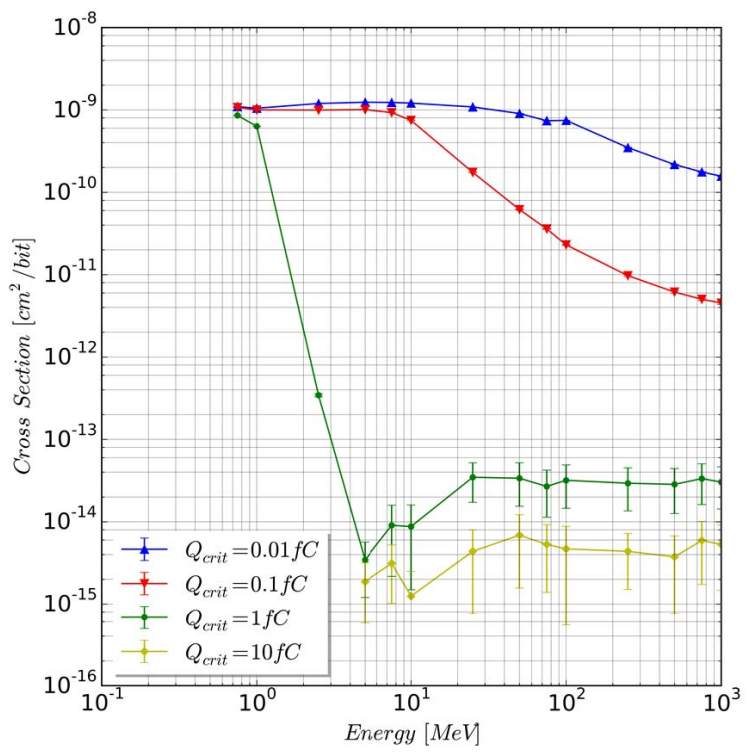

Fig. 5. Evaluation of the SEE cross section due to protons as a function of the incident energy and for different values of the critical charge.

(Fig. 5 and Fig. 6). Once again it is possible to observe how direct ionization from protons (Fig. 5) becomes significant for low critical charges. With regard to muons (Fig. 6), positively and negatively charged muons differ at low energy due to the capture mechanism of the latter: indeed, for critical charges $>\sim 1 \mathrm{fC}$ the contribution of low energy positive muons is negligible.

Three reference environmental cases are reported in the following: $1033 \mathrm{~g} / \mathrm{cm}^{2}$ (ground level) (Fig. 7), $211 \mathrm{~g} / \mathrm{cm}^{2}$ (roughly corresponding to $12 \mathrm{~km}$ altitude) (Fig. 8) and heavy shielding (Fig. 9). Similar results were obtained for the remaining two cases and for which numerical values are provided 


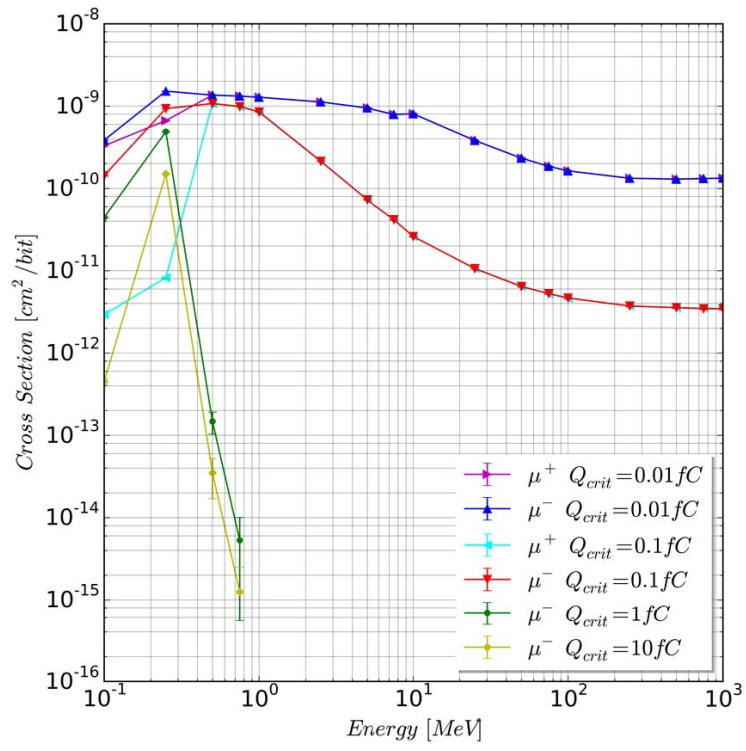

Fig. 6. Evaluation of the SEE cross section due to positive and negative muons as a function of the incident energy and for different values of the critical charge.

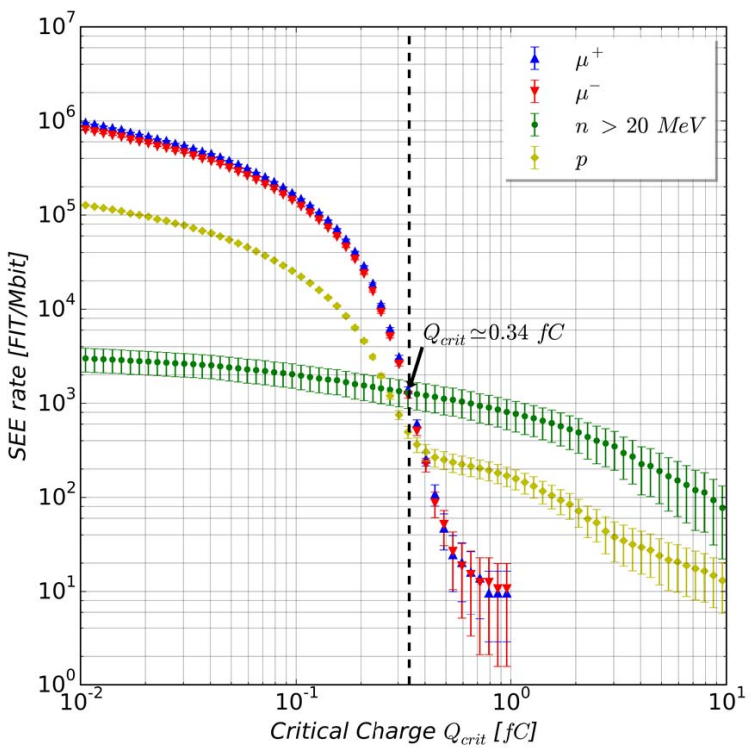

Fig. 7. Evaluation of the SEE rate due to the terrestrial particle environment at $1033 \mathrm{~g} / \mathrm{cm}^{2}$ (ground level) in the area of Vancouver (CA): direct ionization from muon radiation becomes predominant below $\sim 0.34$ fC. Neutron $>20 \mathrm{MeV}$ were considered due to the fall-off the cross section below this energy [30].

in Table I. Fig. 7 shows the expected SEE rate, for the different kind of particle considered in this work, in the $10^{-2}-10^{1} \mathrm{fC}$ critical charge range at ground level. For each data set, a turning point between neutron-charged hadrons/leptons, i.e. the transition $Q_{c r i t}$ below which charged hadrons/leptons dominate, was calculated: as it possible to see from Fig. 7, direct ionization from muon radiation becomes predominant below $\sim 0.34 \mathrm{fC}$. This critical charge was calculated under the hypothesis which our model is based on and must be interpreted as an order of magnitude for the technology it

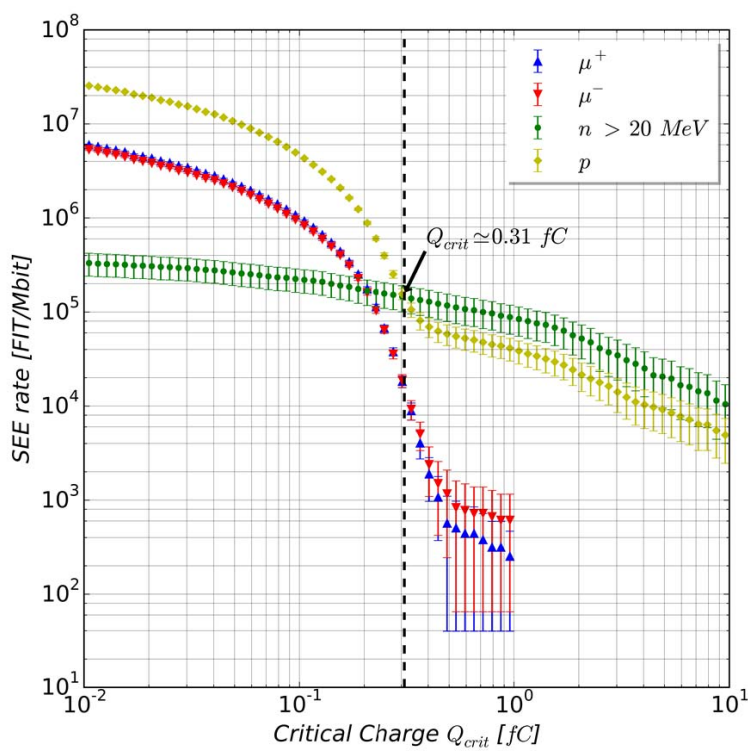

Fig. 8. Evaluation of the SEE rate at $211 \mathrm{~g} / \mathrm{cm}^{2}$ (commercial flight altitude): direct ionization from proton radiation becomes predominant below $\sim 0.31 \mathrm{fC}$.

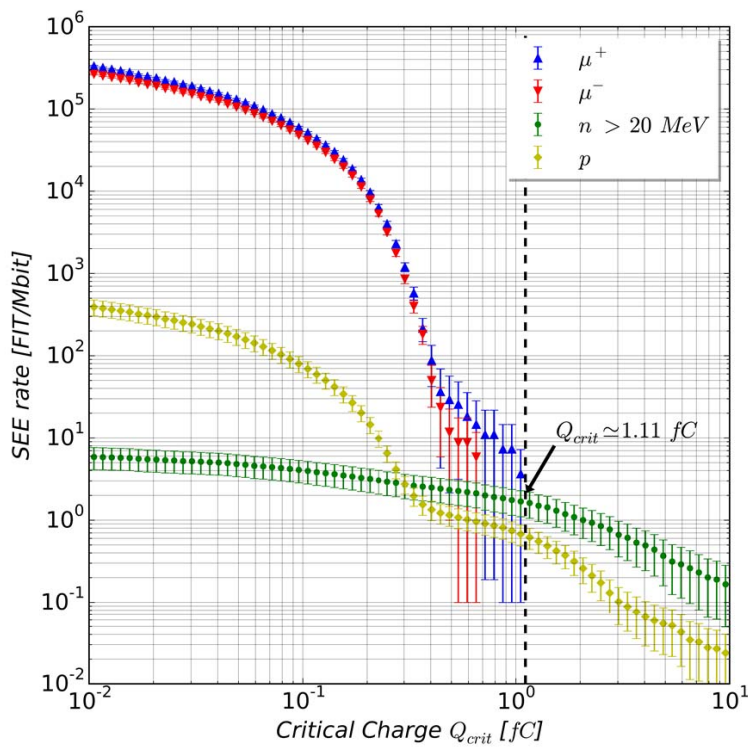

Fig. 9. Evaluation of the SEE rate at $1033 \mathrm{~g} / \mathrm{cm}^{2}$ (ground level) with heavy shielding: direct ionization from muon radiation becomes predominant below $\sim 1.11 \mathrm{fC}$.

TABLE I

Ratio to Ground Level of the Total See Rate at 0.1 AND 1 fC

\begin{tabular}{ccccc}
\hline$Q_{\text {crit }}$ & Heavy Shielding & Light Shielding & $\mathbf{9 0 9} \mathbf{g} / \mathbf{c m}^{\mathbf{2}}$ & $\mathbf{2 1 1} \mathbf{g} / \mathbf{c m}^{\mathbf{2}}$ \\
\hline 0.1 & $0.314 \pm 0.010$ & $1.10 \pm 0.04$ & $1.23 \pm 0.04$ & $21.2 \pm 0.7$ \\
1 & $0.0064 \pm 0.0052$ & $0.29 \pm 0.14$ & $1.9 \pm 0.9$ & $131 \pm 62$ \\
\hline
\end{tabular}

refers to. Sierawski et al., [9] estimated that typical $Q_{\text {crit }}$ for a $45 \mathrm{~nm}$ technology are in the range $0.21-0.71 \mathrm{fC}$ (ground level; terrestrial environment).

Fig. 8 and Fig. 9 show the two extreme cases of the environmental set: $211 \mathrm{~g} / \mathrm{cm}^{2}$ and heavy shielding. In Fig. 8 is possible to see how the contribution of primary and secondary protons dominates the direct ionization range compared to the 


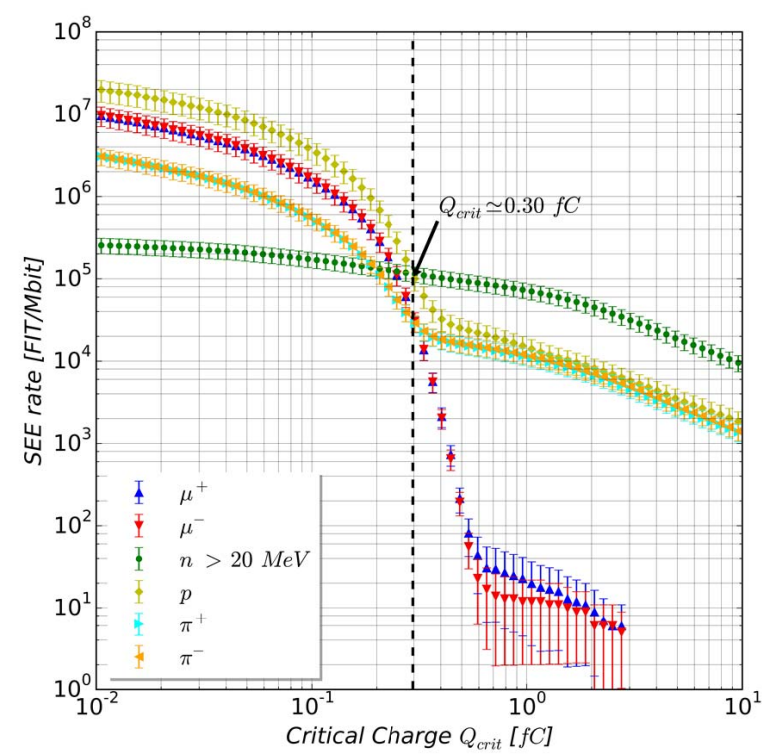

Fig. 10. Evaluation of the SEE rate at the RR alcove: direct ionization from proton radiation becomes predominant below $\sim 0.30 \mathrm{fC}$.

other charged hadrons in a commercial flight environment. On the other hand, in Fig. 9, the contribution of muons, coming from the decay of secondary pions, is predominant and has an increased relative contribution with respect to the ground level case owing to the stronger attenuation of the neutron flux as compared with muons, as reported in [16]. Moreover, a general trend can be identified between the three cases reported above: indeed, by decreasing the atmospheric depth, the dominance of the direct energy deposition moves from protons to muons. The same trend was obtained increasing the thickness of the surrounding shielding. For each case the total SEE rate, i.e. the sum of the expected SEE rate of the particle at a given critical charge, was calculated. To compare different environments we normalize the total SEE rate, for each case studied, to a given reference data set. Table I shows the ratio between the total SEE rate for the $211 \mathrm{~g} / \mathrm{cm}^{2}, 909 \mathrm{~g} / \mathrm{cm}^{2}$, light and heavy shielding data set and the total SEE rate at ground level (which we consider the reference data set for the atmospheric and terrestrial applications) at the reference $Q_{c r i t}$ values of 0.1 and $1 \mathrm{fC}$, representative of the transition between direct and indirect ionization dominance. The ratios reported in Table I show the relative variation of the total SEE rate compared to ground level.

The results of the SEE rate evaluation at the LHC IR7 are shown in Fig. 10 and Fig. 11 for the RR (light shielded) and UJ (heavy shielded) alcove respectively. In both the cases, the direct ionization from protons dominates compared to the other charged hadrons and leptons: indeed, the collimation performed in the warm magnets of the IR7 produces a large background radiation, mainly due to collimation losses. Table II shows the relative variation of the total SEE rate in the light shielded areas (RR) compared to the heavy shielded areas (UJ) at the reference $Q_{\text {crit }}$ values of 0.1 and $1 \mathrm{fC}$.

With regard to the CHARM facility, the detailed evaluation of the SEE rate for the test positions 1, 6 and 11 is reported in Fig. 12 to Fig. 14 respectively, while Table III shows the

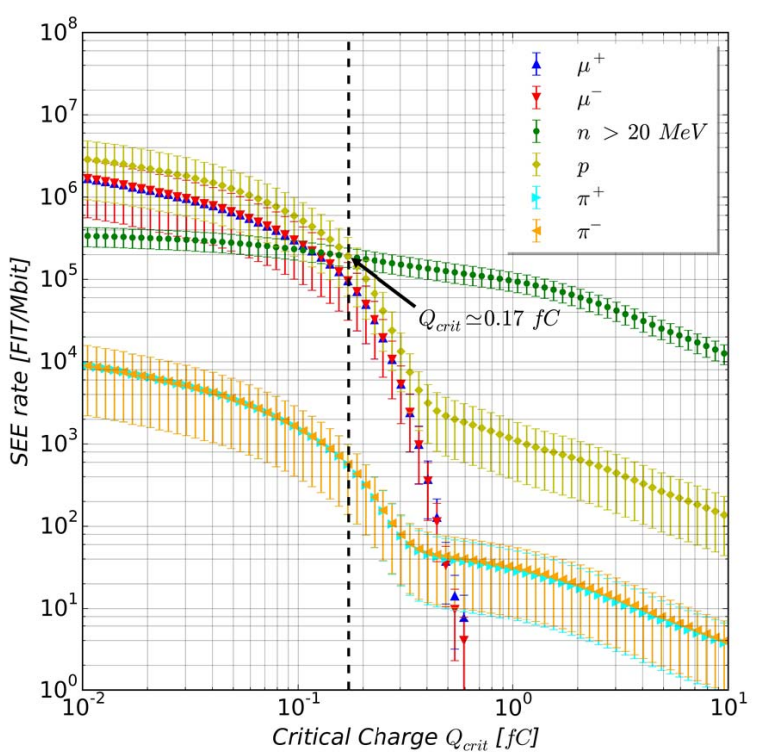

Fig. 11. Evaluation of the SEE rate at the UJ alcove: direct ionization from proton radiation becomes predominant below $\sim 0.17 \mathrm{fC}$.

TABLE II

Ratio of the Total SeE Rate at 0.1 And 1 fC FOR THE LHC IR7 ALCOVES
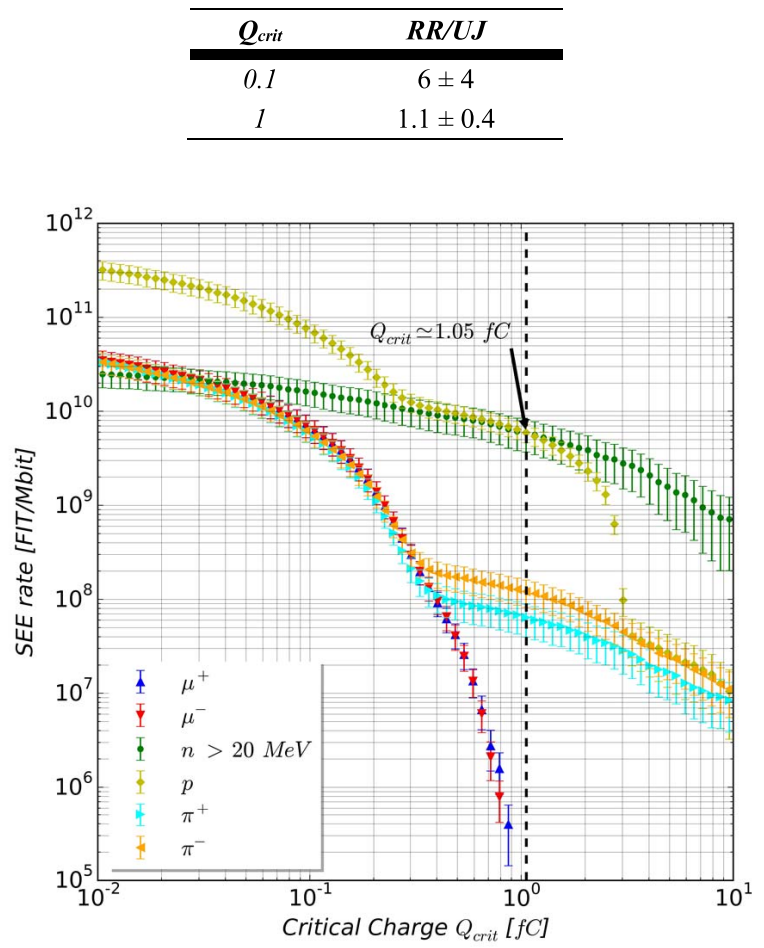

Fig. 12. Evaluation of the SEE rate at the CHARM test position 1: direct ionization from proton radiation becomes predominant below $\sim 1.05 \mathrm{fC}$.

relative variation of the total SEE rate of the test position 1 and 6 (shielded locations) compared to position 11 (unshielded location) at the reference $Q_{c r i t}$ values of 0.1 and $1 \mathrm{fC}$. Figures from Fig. 12 to Fig. 14 show how the SEE rate is indeed proportional to the total HEH fluence, which it is higher in the unshielded test locations downstream the target selector. 
TABLE III

Ratio to the Charm Test Position 11 of the Total SeE Rate at 0.1 AND $1 \mathrm{fC}$

\begin{tabular}{ccc}
\hline $\boldsymbol{Q}_{\text {crit }}$ & CHARM Pos. 1 & CHARM Pos. 6 \\
\hline 0.1 & $0.0068 \pm 0.0017$ & $0.039 \pm 0.004$ \\
1 & $0.021 \pm 0.009$ & $0.058 \pm 0.022$ \\
\hline
\end{tabular}

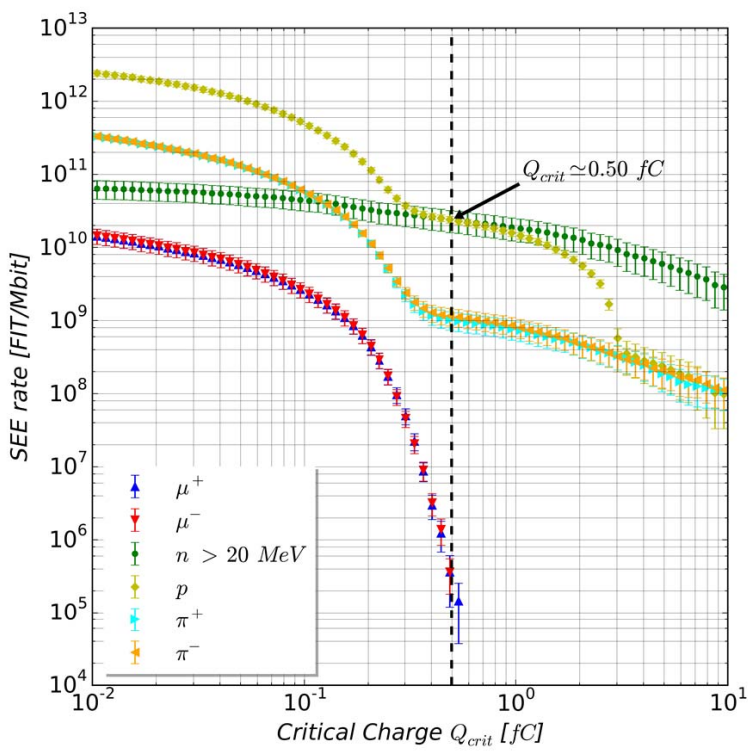

Fig. 13. Evaluation of the SEE rate at the CHARM test position 6: direct ionization from proton radiation becomes predominant below $\sim 0.50 \mathrm{fC}$.

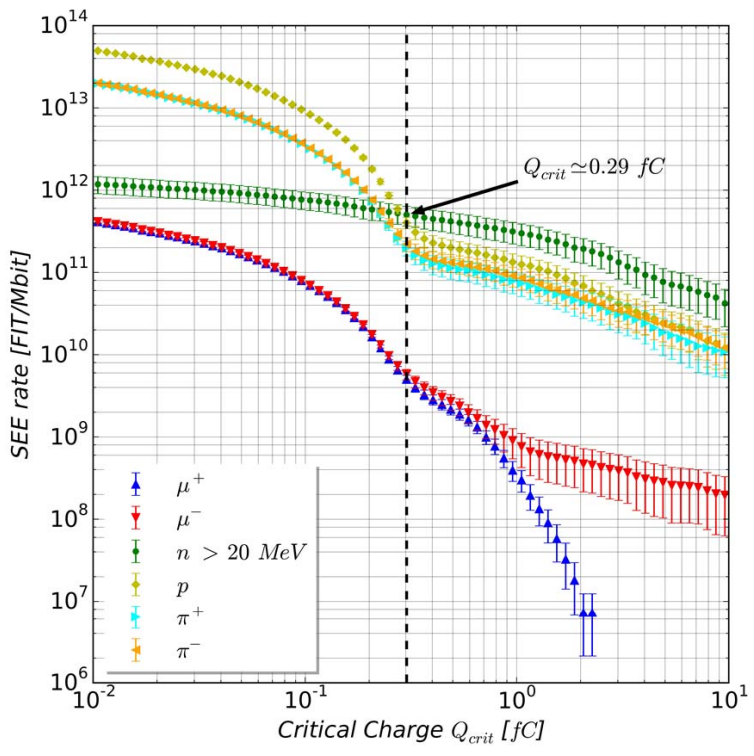

Fig. 14. Evaluation of the SEE rate at the CHARM test position 11: direct ionization from proton radiation becomes predominant below $\sim 0.29 \mathrm{fC}$.

Fig. 15 shows the comparison between the expected total SEE rate in the environmental and in the accelerator cases, studied in this work. To allow a more accurate comparison of the mixed particle energy spectra environments only some reference cases are reported in Fig. $15: 211 \mathrm{~g} / \mathrm{cm}^{2}, 1033 \mathrm{~g} / \mathrm{cm}^{2}$, heavy shielding, LHC-RR alcove, CHARM Position 11. In addition, the different data sets were normalized per unit

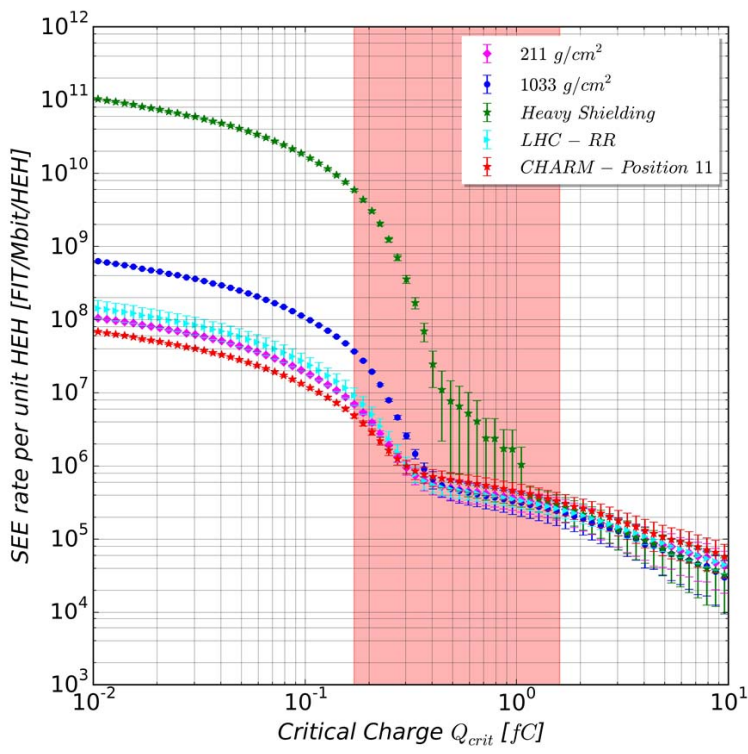

Fig. 15. Comparison of the expected SEE rate between the environmental and the accelerator case. The total SEE curves were normalized per unit HEH fluence, expressed in $\mathrm{cm}^{-2} \mathrm{~s}^{-1}$, in order to be comparable. The critical charge range marked in red represents the region in which the turning point between neutron-charged hadrons/leptons takes place, considering the whole set of data presented in this work.

HEH fluence (hadrons $>20 \mathrm{MeV}$ ) expressed in $\mathrm{cm}^{-2} \mathrm{~s}^{-1}$ since, how already shown above, the SEE rate is indeed proportional to the total HEH fluence.

Fig. 15 shows how the CHARM test position 11 reproduces with a good approximation the SEE rate at flight altitudes and in the LHC-RR alcoves, particularly above $\sim 0.3-0.4 \mathrm{fC}$. Comparing the curves at $0.1 \mathrm{fC}$ the agreement is within a factor 2 considering the RR alcoves and within a factor 1.5 considering flight altitudes. With regard to the ground level applications, again CHARM is able to reproduce the SEE rate curve within the statistical uncertainties above $\sim 0.3-0.4 \mathrm{fC}$; at $0.1 \mathrm{fC}$ the agreement is within a factor 8 . The heavy shielding case, at ground level, is no longer reproduced below $\sim 1 \mathrm{fC}$. The critical charge range marked in red represents the region in which, considering the whole set of cases studied in this work, the turning point between neutron-charged hadrons/leptons dominance takes place. Once again, this critical charge range was obtained under the hypothesis which our model is based on and must be intended as an order of magnitude for the technology it refers to.

Fig. 15 can be a useful reference in planning new accelerator facility or in the design of new technologies for specific applications, which require low critical charges. Indeed, even if the present work is focused on the simulation side and no experimental measurements have been conducted yet, FLUKA was already validated in the past in similar studies as reported in [21], [24], [31] showing an excellent agreement between simulations and experimental measurements. Table IV shows the ratio, for each data set, of the expected total SEE rate between 0.1 and $1 \mathrm{fC}$. Due to the trend in scaling toward smaller integrated circuit feature sizes, which translates in lower critical charges, Table IV gives a first indication of the SEE rate in the future technology. 
TABLE IV

Ratio of the Expected Total SeE Rate Between 0.1 And 1 fC

\begin{tabular}{cc}
\hline Data Set & Ratio 0.1/1 fC \\
\hline Heavy Shielding & $15000 \pm 11000$ \\
Light Shielding & $1200 \pm 400$ \\
$1033 \mathrm{~g} / \mathrm{cm}^{2}$ & $310 \pm 110$ \\
$909 \mathrm{~g} / \mathrm{cm}^{2}$ & $200 \pm 70$ \\
$211 \mathrm{~g} / \mathrm{cm}^{2}$ & $51 \pm 17$ \\
LHC RR & $71 \pm 25$ \\
LHC UJ & $13 \pm 9$ \\
CHARM 1 & $9 \pm 7$ \\
CHARM 6 & $18 \pm 8$ \\
CHARM 11 & $27 \pm 4$ \\
\hline
\end{tabular}

\section{CONCLUSIONS}

In this work, the expected SEE rate in a general model of a state-of-the-art SRAM memory was studied as a function of the critical charge in different radiation environments. Compared to other published work [15], we used a full Monte Carlo approach for the assessment of both monoenergetic cross sections and SEE rate as well as the primary particle spectra in different radiation environments. FLUKA Monte Carlo simulation was used to study the energy deposition in the SRAM model and to transport particle spectra previously assessed in different radiation environments such as the terrestrial environment, commercial flight altitude, LHC critical areas for electronics and CERN's CHARM test facility.

Mono-energetic cross section for protons and muons were evaluated in the $0.1-1000 \mathrm{MeV}$ energy range. Results show how components that are sensitive to low energy electrons may be sensitive to direct ionization by high energy protons as well. Muon capture becomes a significant source of induced SEE at low energy while at high energy positive and negative muons interact in the same way with the sensitive volume.

With regard to the mixed particle energy spectra study, a detailed analysis of the contribution of different particles to the total SEE rate was performed for the different radiation environments. Considering the environmental field, the turning point between indirect-direct ionization was calculated to be of the order of magnitude of $\sim 0.3 \mathrm{fC}$ at flight altitudes and ground level ( $\sim 1.1 \mathrm{fC}$ considering a generic heavy shielding). Direct ionization at flight altitudes is dominated by primary and secondary protons produced in the interaction of GCR with the Earth's atmosphere; at ground level, muons, which are the most numerous species, represent the most important contribution to direct ionization. In the LHC environment, protons coming from beam losses and secondary interactions represent the most important contribution in the direct ionization together with muons produced from pion's decay. In RR alcoves, the turning point was calculated to be $0.3 \mathrm{fC}$; in UJ alcoves, heavily shielded compared to RR, the turning point was calculated to be $0.17 \mathrm{fC}$. With regard to the CHARM facility, three different test locations were studied: the direct ionization is dominated from protons produced in secondary interactions. The turning point varies from $\sim 1 \mathrm{fC}$ of the lateral position 1 to $\sim 0.3 \mathrm{fC}$ of the position 11 , downstream the target selector.
Finally the environmental and the accelerator field, both LHC and CHARM, were compared: considering all the data sets, the critical charge $Q_{\text {crit }}$ of the turning point was calculated to be in the range $\sim 0.2-1.1 \mathrm{fC}$. Comparing the total SEE rate per unit HEH fluence is possible to see how CHARM is able to reproduce within a factor 2 both the flight altitudes and LHC RR alcoves, as well as ground level applications above $\sim 0.3-0.4 \mathrm{fC}$. This confirms how accelerator-driven studies can be used to design, test and improve the future technology of interest in avionics, space and ground level applications.

\section{REFERENCES}

[1] J. F. Ziegler, "Terrestrial cosmic rays," IBM J. Res. Develop., vol. 40, no. 1, pp. 19-39, Jan. 1996.

[2] E. Normand, "Single-event effects in avionics," IEEE Trans. Nucl. Sci., vol. 43, no. 2, pp. 461-474, Apr. 1996.

[3] E. Normand, "Single event upset at ground level," IEEE Trans. Nucl. Sci., vol. 43, no. 6, pp. 2742-2750, Dec. 1996.

[4] P. E. Dodd and L. W. Massengill, "Basic mechanisms and modeling of single-event upset in digital microelectronics," IEEE Trans. Nucl. Sci., vol. 50, no. 3, pp. 583-602, Jun. 2003.

[5] Measurement and Reporting of Alpha Particle and Terrestrial Cosmic Ray-Induced Soft Errors in Semiconductor Devices, JEDEC Standard 89A (JESD89A), JEDEC Solid State Technology Association, Arlington, VA, USA, Oct. 2006.

[6] J. F. Ziegler, H. P. Muhlfeld, C. J. Montrose, H. W. Curtis, T. J. O'Gorman, and J. M. Ross, "Accelerated testing for cosmic soft-error rate," IBM J. Res. Develop., vol. 40, no. 1, pp. 51-72, Jan. 1996.

[7] B. D. Sierawski et al., "Muon-induced single event upsets in deepsubmicron technology," IEEE Trans. Nucl. Sci., vol. 57, no. 6, pp. 3273-3278, Dec. 2010.

[8] S. Semikh, S. Serre, J. L. Autran, D. Munteanu, G. Gasiot, and P. Roche, "Effects of low-energy muons on electronics: Physical insights and Geant4 simulations," in Proc. 13th Eur. Conf. Radiat. Effects Compon. Syst. (RADECS), Biarritz, France, Sep. 2012.

[9] B. D. Sierawski et al., "Effects of scaling on muon-induced soft errors," in Proc. IEEE Int. Rel. Phys. Symp. (IRPS), Monterey, CA, USA, Apr. 2011, pp. 3C.3.1-3C.3.6.

[10] N. Seifert, S. Jahinuzzaman, J. Velamala, and N. Patel, "Susceptibility of planar and 3D tri-gate technologies to muon-induced single event upsets," in Proc. IEEE Int. Rel. Phys. Symp. (IRPS), Monterey, CA, USA, Apr. 2015, pp. 2C.1.1-2C.1.6.

[11] F. Lei, S. Clucas, C. Dyer, and P. Truscott, "An atmospheric radiation model based on response matrices generated by detailed Monte Carlo simulations of cosmic ray interactions," IEEE Trans. Nucl. Sci., vol. 51, no. 6, pp. 3442-3451, Dec. 2004.

[12] F. Lei, A. Hands, S. Clucas, C. Dyer, and P. Truscott, "Improvement to and validations of the QinetiQ atmospheric radiation model (QARM)," IEEE Trans. Nucl. Sci., vol. 53, no. 4, pp. 1851-1858, Aug. 2006.

[13] T. Sato and K. Niita, "Analytical functions to predict cosmic-ray neutron spectra in the atmosphere," Radiat. Res., vol. 166, no. 3, pp. 544-555, 2006.

[14] T. Sato, H. Yasuda, K. Niita, A. Endo, and L. Sihver, "Development of PARMA: PHITS-based analytical radiation model in the atmosphere," Radiat. Res., vol. 170, no. 2, pp. 244-259, 2008.

[15] G. Hubert, L. Artola, and D. Regis, "Impact of scaling on the soft error sensitivity of bulk, FDSOI and FinFET technologies due to atmospheric radiation," Integr. VLSI J., vol. 50, pp. 39-47, Jun. 2015. [Online]. Available: http://dx.doi.org/10.1016/j.vlsi.2015.01.003

[16] A. Infantino, E. W. Blackmore, M. Brugger, R. G. Alía, M. Stukel, and M. Trinczek, "FLUKA Monte Carlo assessment of the terrestrial muon flux at low energies and comparison against experimental measurements," Nucl. Instrum. Methods, Phys. Res. A, vol. 838, pp. 109-116, 2016.

[17] B. Bellesia et al., "Impacts of SEEs," in Proc. LHC Perform. Workshop, Chamonix, France, 2009, pp. 160-170.

[18] G. Battistoni et al., "The application of the Monte Carlo code FLUKA in radiation protection studies for the large hadron collider," Prog. Nucl. Sci. Technol., vol. 2, pp. 358-364, 2011. 
[19] K. Roed et al., "FLUKA simulations for SEE studies of critical LHC underground areas," IEEE Trans. Nucl. Sci., vol. 58, no. 3, pp. 932-938, Jun. 2011

[20] A. Thornton, "CHARM facility test area radiation field description," CERN, Geneva, Switzerland, Tech. Rep. CERN-ACC-NOTE-201612345, 2016.

[21] R. G. Alía et al., "SEL hardness assurance in a mixed radiation field," IEEE Trans. Nuc. Sci., vol. 62, no. 6, pp. 2555-2562, Dec. 2015.

[22] T. T. Böhlen et al., "The FLUKA code: Developments and challenges for high energy and medical applications," Nucl. Data Sheets, vol. 120, pp. 211-214, Jun. 2014.

[23] A. Ferrari, P. R. Sala, A. Fassò, and J. Ranft, document CERN-2005-10, INFN/TC_05/11, SLAC-R-773, 2005.

[24] R. G. Alia et al., "SEU measurements and simulations in a mixed field environment," IEEE Trans. Nucl. Sci., vol. 60, no. 4, pp. 2469-2476, Aug. 2013.

[25] M. J. Gadlage, A. H. Roach, A. R. Duncan, M. W. Savage, and M. J. Kay, "Electron-induced single-event upsets in 45-nm and 28-nm bulk CMOS SRAM-based FPGAs operating at nominal voltage," IEEE Trans. Nucl. Sci., vol. 62, no. 6, pp. 2717-2724, Dec. 2015.
[26] E. W. Blackmore, M. Stukel, M. Trinczek, C. Slayman, S.-J. Wen, and R. Wong, "Terrestrial muon flux measurement at low energies for soft error studies," IEEE Trans. Nucl. Sci., vol. 62, no. 6, pp. 2792-2796, Dec. 2015.

[27] K. Roeed, M. Brugger, and G. Spiezia, "An overview of the radiation environment at the LHC in light of R2E irradiation test activities," CERN, Geneva, Switzerland, Tech. Rep. CERN-ATS-Note-2011-077, 2012.

[28] H. H. K. Tang, "Nuclear physics of cosmic ray interaction with semiconductor materials: Particle-induced soft errors from a physicist's perspective," IBM J. Res. Develop., vol. 40, no. 1, pp. 91-108, Jan. 1996.

[29] J. M. Trippe et al., "Electron-induced single event upsets in $28 \mathrm{~nm}$ and $45 \mathrm{~nm}$ bulk SRAMs," IEEE Trans. Nucl. Sci., vol. 62, no. 6, pp. 2709-2716, Dec. 2015.

[30] K. Roeed et al., "Method for measuring mixed field radiation levels relevant for SEEs at the LHC," IEEE Trans. Nucl. Sci., vol. 59, no. 4, pp. 1040-1047, Aug. 2012.

[31] J. Mekki et al., "CHARM: A mixed field facility at CERN for radiation tests in ground, atmospheric, space and accelerator representative environments," IEEE Trans. Nucl. Sci., vol. 63, no. 4, pp. 2106-2114, Aug. 2016. 